\title{
Data Visualization on Crime against Women
}

\author{
Jaya Preethi Mohan, Dr.D.Usha
}

\begin{abstract}
Visualization ensures the modern expectation of all forms of data. It is important to understand the data and its statistical variance graphically. Visualization on crime data would be supportive to analyze and prevent the threats in society. According to recent surveys and records, India has undergone many crime issues which occur on women. In order to prevent and analyze the crime issues against women, Data visualization is a useful approach to deal with it. The current data technologies available are appropriate to accomplish the task of visualization for women safety. Efficient visualization with effective machine learning algorithm and its performance finds the response for data related requests in the field of data science. This paper clarifies the details of crime against women through a graphical approach and illustrates about how to notify the unsafe levels by alert to safeguard the women.
\end{abstract}

Index Terms: algorithm, machine learning, visualization, women safety

\section{INTRODUCTION}

Though the world has seen enormous developments in all the sectors, it faces crime issues all-time. Research results discovered many technologies and findings to analyze criminal cases. In this digital era, data plays a vital role to depict the crime details clearly. It is mandatory to collect and maintain the crime data for present and future purposes which helps for analysis and analytics. Prediction on the crime scene will not end up in accuracy but its analysis result will create awareness about the future consequences. Safety brings responsibility to each and every person. It is indeed that women and children are the high victims of crime scene happen everywhere. Although feminism spreads widely in society, the crime against women increases day by day. As a result of prediction and analysis on crime rate, data analysis with visualization also helps well for detecting threats.

\section{II.ALGORITHM ON CRIME ANALYSIS}

\section{A. Purpose of using algorithms on crime analysis}

Machine learning algorithms are powerful to find the probabilities. It is possible to find the uncertainty and forecasting values of an existing data with the help of the probability values. Each and every day the rate of crime records increases drastically. Data Science and Machine

Learning supports the investigation process by analyzing the data. Once the data has been analyzed, it should be able to show the values in range. Bulgary, arson and other National Crime Records Bureau Crimes have been dropped down but

Revised Manuscript Received on July 05, 2019.

Jaya Preethi Mohan, Computer Science and Engineering, Dr.M.G.R. Educational and Research Institute, Chennai, India.

Dr.D.Usha, Computer Science and Engineering, Dr.M.G.R. Educational and Research Institute, Chennai, India. sexual abuse, rape, murder cases have been goes up statistically. Predicting the victims of crime are unpredictable but place of crime occurrences, criminal's attributes; cause of crime is predictable (1).

\section{B. Importance of visualizing the existing crime data}

Criminal misuses technology to indulge in crime activities but investigation team uses technology to block those activities. Data Visualization is a promising concept of data science which represents the data in a graphical format. Clear understanding on data ranging is highly important to get those value in right. Visual representations are useful to identify the statistical changes in a short span of time (2).Using machine learning algorithm for data visualization will identify the insights of the data visually..

\section{FACTOR VECTOR VISUALIZATION}

Fractional Vector Visualization (FVV) Algorithm is a supervised learning algorithm of machine learning. FVV is a pointed weapon which chops the data into fragments of n-dimensional space like Support Vector Machine. It is perfect for using in classification problems of small dataset which has already analyzed by regression and logistic models. Each data has plotted by dividing the plot into multiple segments where FVV identifies the insight of the specific data. During every attempt of implementation, it assigns new input or output to get another input or output value. Those I/O value represents as example in n-dimensional space with visualization

\section{A. FVV formulation from SVM}

SVM uses the hypothesis spaces and loss functions. Optimal hyperplane is the solution of the learning problem in SVM's folklore view. The input data $x$ lies on the space of the hyperplane lies. The simplest formulation of SVM is the linear one, where hyperplane becomes the subset of all the forms. For each class, the margin connecting the support planes is simplified as below. $\mathrm{x}$ is the new data point of the classification function.

$$
f(x)=w \cdot x+b
$$

it is formulated as

$$
\begin{gathered}
\text { Min } \Psi(w, b, z)=(1 / 2)\|w\| 2+C \sum=\text { mi } i z \\
\text { s.t. } y i(w . x i-b) \quad+z i \geq 1 \\
z i \geq 0(i=1, \ldots, m)
\end{gathered}
$$

$z i \geq 0$ is slack variable, $C>0$ is constant used to refrain errors and margin size. The 2-norm of the vector $w$ is $\|w\| .(i=1, \ldots$, $m$ ) are the data points of the $n$-dimensional input space. 


\section{B. SVM and visualization as FVV}

1) The modern visualization methods indulge deeply in visual data mining techniques.

2) $2 \mathrm{D}$ scatter plots and the parallel coordinates are the two common visualization techniques used for data exploration

3) The nonlinear methods of machine learning are flexible and results in generalization, when compared to common classical methods of statistics which is still considered as black box models by the users.

4) Black-box model visualization and interpretation follows standard visualization techniques to project high-dimensional data for 2D plots and plane which may approximate SVM model like a decision tree rules

5) Rule Extraction and Visualization are the two major phases of black-box models.

6) If ... then ...else rules are obtained by the method of Rule Extraction from SVM model.

7) Visualization of vector model identifies the approximate of 2-D plane with a variable formation in the projected 2-D Space.

8) It occurs by depends on the location of a support vector and the fraction of a data in the projected 2-D space (5).

\section{Application}

1) Fractional Vector Visualization algorithm is used to find the generalized predictive Control of variables with data classification

2) Strengthens the Geo-Spatial analysis by fragmenting the data coordinates and also for the identification of geo location.

3) Image classification, face recognition methods uses support vector machine for matching the data points and processing it also majorly useful for text classification especially in natural language processing.

4) Health care researchers may apply support vector machine oriented algorithm like FVV for finding the cell deviation which is helpful in disease detection

5) It cut downs the seconds to milliseconds and brings out the minute changes in the time series (3).

\section{SVM Graphical Representation}

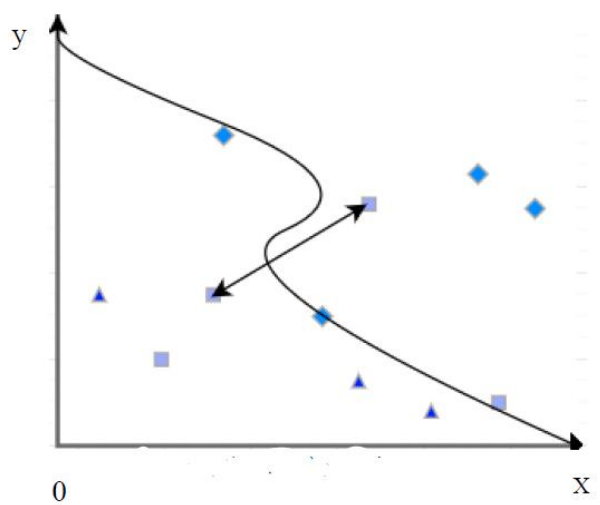

Fig 1: I/O in SVM

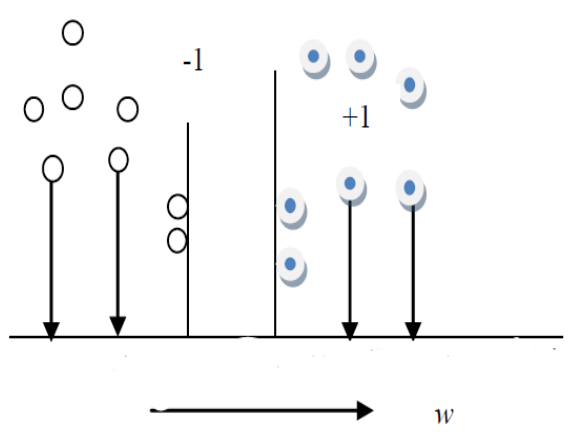

Figure 2: FVV formulation from FVV

\section{FVV ON CRIME AGAINST WOMEN}

Data mining and visualization algorithm have used to analyze the data of crime against women in many research diaries. After the analysis, it is important to know the fractional deviation of the analyzed data which showcases the probabilities of crime instance as vectors. Illustration of FVV in the crime data is useful to understand the variations of data and its performances. By knowing this information on the data divisions in multi dimensional space results in alert to the unsafe levels which are mandatory to safeguard the women.

\section{A. Data Segregation}

The data has been mined from National Crime Records Bureau and segregated according to the needs of machine learning code. The various parameters were examined from the raw data and extracted the required parameter for Vector visualization.

\section{B. Visualization of data attributes}

The number of cases recorded to the victim's age level has been visualized using Factional Vector Visualization.
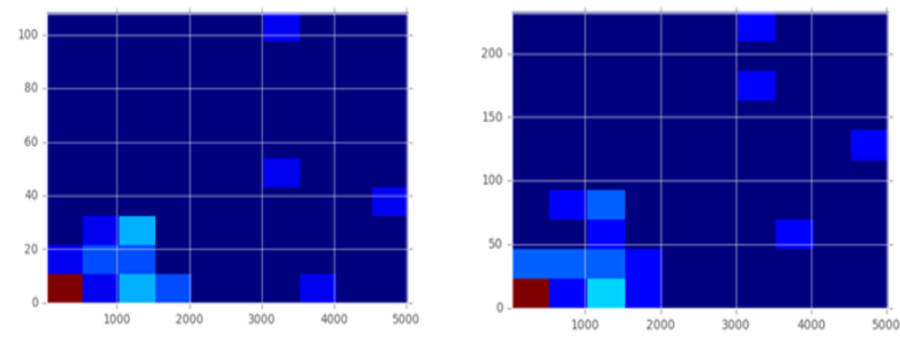

Fig 3: Below 6 years

Fig 4: Above 6 years below 12 years

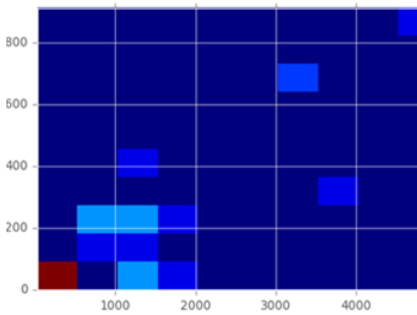

Fig 5: Above 12- below 16 years

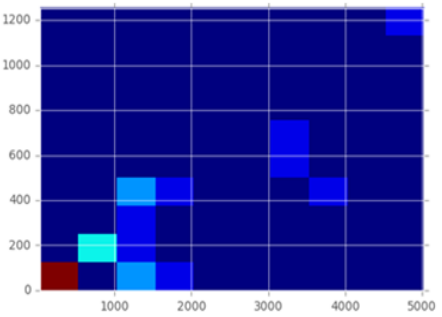

Fig 6: Above 6 - below 12 years 


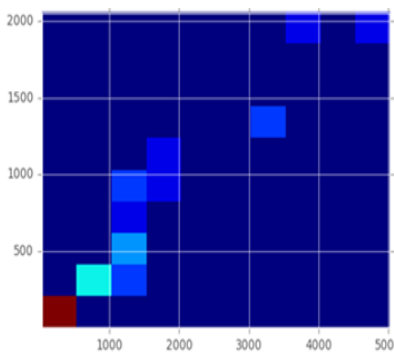

Fig 7: Above 18- below 30 years

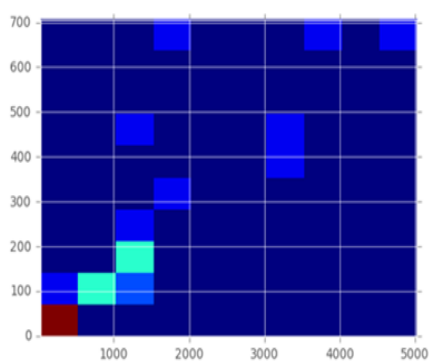

Fig 8: Above 30 - below 45 years

\section{CONCLUSION}

The research on data visualization on crime against women results in suggestion to use Fractional Vector Visualization for crime analysis. This is highlighted for the specific crime issues like crime against women cases. The mining of real data with exact values would be helpful in achieving the accuracy. Merging the concept of visualization and machine learning for a crime investigation purpose accomplishes the best outcome for findings and decision making.

\section{REFERENCES}

1. Sathyadevan, Shiju \& S, Devan \& S Gangadharan, Surya. (2014). Crime Analysis and Prediction Using Data Mining. 10.1109/CNSC.2014.6906719.

2. Tushar Sonawanev, Shirin Shaikh, Shaista Shaikh, Rahul Shinde, Asif Sayyad Vol-1 Issue-4 2015. Crime analysis Prediction and Visualization using data mining. IJARIIE-ISSN(O)-2395-4396

3. Theodoros Evgeniou and Massimiliano Pontil Center for Biological and Computational Learning, and Artificial Intelligence Laboratory, MIT, E25-201, Cambridge, MA 02139.

4. Do, Thanh-Nghi \& Poulet, François. (2004). Enhancing SVM with Visualization. 3245. 183-194. 10.1007/978-3-540-30214-8_14.

5. Sauptik Dhar and Vladimir Cherkassky, Department of Electrical and Computer Engineering, Universityof Minnesota, Minneapolis MN 55455 USA.

6. Caragea, Doina \& Cook, Dianne \& Wickham, Hadley \& Honavar, Vasant. (2008). Visual Methods for Examining SVM Classifiers. 10.1007/978-3-540-71080-6_10.

7. Maszczyk, Tomasz \& Wlodzislaw, Duch. (2008). Support Vector Machines for Visualization and Dimensionality Reduction. 5163. 346-356. 10.1007/978-3-540-87536-9_36. 